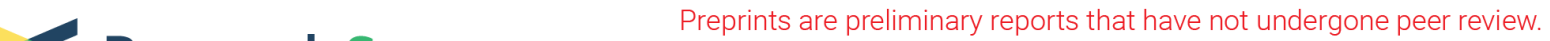 Research Square
or referenced by the media as validated information.
}

\section{The impact of combining dental interventions with dietary and exercise advice on waist circumference in patients with the metabolic syndrome: Protocol for a randomized controlled trial}

\section{Hiroyuki Suzuki}

Gerodontology and Oral Rehabilitation, Graduate School of Medical and Dental Sciences, Tokyo Medical and Dental University

Yuriko Komagamine ( $\nabla$ y.komagamine.gerd@tmd.ac.jp)

Tokyo Medical and Dental University

\section{Manabu Kanazawa}

Gerodontology and Oral rehabilitation, Graduate School of Medical and Dental Sciences, Tokyo Medical and Dental University

\section{Maiko Iwaki}

General Dentistry, Graduate School of Medical and Dental Sciences, Tokyo Medical and Dental University

\section{Midori Doke}

Gerodontology and Oral Rehabilitation, Graduate School of Medical and Dental Sciences, Tokyo Medical and Dental University

\section{Tetsuya Mizuno}

Laboratory of Health Science and Physical Education, College of Liberal Arts and Science, Tokyo Medical and Dental University

\section{Manabu Sema}

Respiratory Medicine, Graduate School of Medical and Dental Sciences, Tokyo Medical and Dental University

\section{Kaori Okayasu}

Respiratory Medicine, Yokohama City Minato Red Cross Hospital

\section{Yasunari Miyazaki}

Respiratory Medicine, Graduate School of Medical and Dental Sciences, Tokyo Medical and Dental University

\section{Shunsuke Minakuchi}

Gerodontology and Oral Rehabilitation, Graduate School of Medical and Dental Sciences, Tokyo Medical and Dental University 


\section{Study protocol}

Keywords: Metabolic Syndrome, Prosthetic treatment, Periodontal treatment, Dietary advice, Exercise advice, Waist circumference

Posted Date: September 18th, 2019

DOI: https://doi.org/10.21203/rs.2.479/v2

License: (c) (i) This work is licensed under a Creative Commons Attribution 4.0 International License.

Read Full License 


\section{Abstract}

Background Metabolic Syndrome (MetS) associated with cardiovascular diseases have been focused on by researchers. Although various studies have investigated its relationship with the oral environment, the effects of dental interventions for MetS remain unclear. The aim of this trial is to investigate whether prosthetic or periodontal treatment combined with dietary and exercise advice can improve waist circumference, a surrogate measure of the syndrome. The null hypothesis is that there will be no difference in waist circumference between participants who receive prosthetic or periodontal treatment combined with dietary and exercise advice and those who receive only dietary and exercise advice. Methods This is a single-center, single-blinded, parallel, randomized controlled trial. Participants are randomly allocated to an intervention group (dietary and exercise advice plus prosthetic or periodontal treatment) or a control group (dietary and exercise advice only). Outcome measures include waist circumference; blood pressure; serum triglyceride, serum HDL/LDL cholesterol, fasting blood glucose, and $\mathrm{HbA1c}$ assessment using blood tests; Anthropometric measurements using bioelectrical impedance; health-related quality of life assessment using MOS 36-Item Short-Form Health Survey; oral-health-related quality of life assessment using Oral Health Impact Profile-14 and Geriatric Oral Health Assessment Index; food and nutrient intake assessment using brief-type self-administered diet history questionnaire; periodontal assessment using probing pocket depth and bleeding on probing. Objective masticatory function assessment using a color-changeable chewing gum and a gummy jelly was conducted for the participants who received prosthetic treatment. Outcome measures are to be implemented at three time points: at baseline and at 1 and 3 months after intervention. Differences between two groups in each assessment is compared using the Student's t-test. Within group, the change between each assessment time is compared using the Tukey's test. Discussion This trial could help to establish a new preventive method for the MetS that can be delivered by dental practitioners. Moreover, the findings are expected to reinforce the contribution of dentistry to extending healthy life expectancy.

\section{Background}

The World Health Organization focused on the prevention of cardiovascular diseases in the World Health Report 2002 [1]. In Japan, the Ministry of Health, Labor and Welfare has reported that over $50 \%$ of mortality is caused by lifestyle-related diseases, including cardiovascular disease, diabetes mellitus, and malignant neoplasia [2]. One cause of cardiovascular disease, metabolic syndrome (MetS), has recently become a clinical focus. MetS is defined as a complex state of increasing waist circumference, blood triglyceride levels, blood glucose levels, and blood pressure that occurs due to the accumulation of visceral fat [3]. Early detection and treatment of MetS is considered important in the prevention and progression of cardiovascular diseases.

Several studies have investigated the relationship between MetS and the oral environment. The relationships between tooth loss and mortality in diabetes mellitus and arteriosclerotic disease have already been reported. Nowjack-Raymer et al. [4] reported that reduced vegetable intake caused by tooth loss. The reduction of vegetable and fruit intake was a risk factor for the onset of both cardiovascular 
disease and diabetes mellitus [5,6]. Moreover, cross-sectional research by Patel et al. [7] indicated that patients with diabetes mellitus who were older than 50 years had more missing teeth than healthy peers and were 2.25 times more likely to be edentulous. Schwahn et al. [8] also reported that having nine or more unreplaced teeth was related to all-cause and cardiovascular mortality (rate ratios of 1.43 and 1.88, respectively). Thus, tooth loss appears to be related to mortality in patients with cardiovascular disease and diabetes mellitus.

In research by Zhu et al. [9], it was reported that the number of natural teeth was inversely associated with body mass index, waist circumference, blood pressure, fasting plasma glucose, and insulin concentration. In the same research, it was positively associated with high-density lipoprotein cholesterol concentrations. This report therefore suggested an inverse association between the number of natural teeth and the presence of MetS. Elsewhere, there have been several reports $[10,11]$ indicating a significant correlation between periodontitis and MetS, such as that by Kaye et al. [11] who reported a relationship to disease progression.

In a randomized controlled trial, Bradbury et al. [12] revealed that new complete denture fabrication and tailored dietary counseling could improve fruit and vegetable intake in edentulous elderly people. Other randomized controlled trials $[13,14]$ have also shown that fabricating new complete dentures and giving simple dietary advice by patient information leaflet can improve the quality of food and nutrient intake. Although these suggest that combining prosthetic treatment with dietary advice can improve food and nutrient intake, the effects on MetS have not been clarified. There are also conflicting reports about the effect of non-surgical periodontal treatment on hemoglobin A1c (HbA1c) in type 2 diabetes mellitus. On the one hand, Hayashi et al. [15] reported that non-surgical periodontal treatment could improve HbA1c, but on the other hand, Mizuno et al. [16] reported that it did not. The effects on MetS are also unclear.

Treatments for MetS include diet therapy, kinesitherapy, and lifestyle improvements (e.g., smoking cessation). However, little is known of the effect of dental interventions (e.g. prosthetic or periodontal treatment) combined with dietary and exercise advice on MetS outcome measures like waist circumference. We therefore aim to compare this outcome between an intervention group (dental interventions plus diet and exercise advice) and a control group (diet and exercise advice only). Our null hypothesis is that there will be no difference in waist circumference between the intervention and control groups.

\section{Methods/design}

\section{Trial design}

We plan to conduct a single-centered, single-blinded, parallel, randomized controlled trial according to the 2010 Consolidated Standards of Reporting Trials (CONSORT) [17]. The trial protocol and informed consent documents have been approved by the ethics committee of the Faculty of Dentistry, Tokyo Medical and Dental University (TMDU), registry number D2016-028. This trial is also registered with the University Hospital Medical Information Network Center (Unique Trial Number: UMIN000022753). 
All participants will be required to provide written informed consent before participating. They will then be randomly allocated to the intervention group or the control group. If the control group participants require prosthetic or periodontal treatment, it will be performed at the end of the trial.

\section{Participants}

This trial will be conducted among the staff and outpatients of the TMDU Hospital of Medicine and Dentistry. We will recruit participants through recruitment documents and flyers. Recruitment documents will be sent to all TMDU staff who undergo periodic health examination, and the responders will be enrolled. These documents include a statement that participation is voluntary and not a requirement of their daily work. Flyers will also be posted in the reception room of the TMDU Hospital of Medicine and Dentistry, and responder will be enrolled. Contracted companies will be partially responsible for overseeing participant recruitment. All participants need to meet the eligibility criteria.

\section{Eligibility criteria}

First, participants will undergo screening to check that they meet the inclusion criteria for the trial, which will be conducted by an outcome examiner (MD). Next, we will record the participants' ages, genders, comorbidities, and medications. Oral examination and radiography will be performed to diagnose the degree of periodontal disease and the presence (or number) of missing teeth. Preliminary treatment will be given, as needed, for dental caries (including root canal treatment) before the intervention phase.

Each participant will be required to meet the following inclusion criteria: the Japanese waist circumference requirement for MetS [18]; Eichner Index [19] B1-4, C1-3 and/or slight-to-moderate periodontitis [20]; over 40 years old; no medication change for 3 months; and who provide written informed consent. In addition, participants will be excluded if they meet the following criteria: regularly receive dietary or exercise counseling (e.g., by a nutritionist); have any dietary restriction due to a health problem; have no means to implement dietary change (e.g., institutionalized); medication has been changed in the last 3 months; have serious heart disease, hypothyroidism or hyperthyroidism, severe liver impairment, severe kidney impairment, or a severe psychiatric disorder; are undergoing cancer therapy; have experienced significant weight loss during the last 3 months; are pregnant; or are deemed ineligible by the responsible persons in this trial (MK).

\section{Interventions}

Figure 1 shows the trial flow diagram. After baseline evaluation, all participants will receive dietary and exercise advice using the Total Fitness Analysis System (TFAS) [21]. Participants who have missing teeth or periodontitis in the intervention group will then receive prosthetic or periodontal treatment.

\section{Dietary and exercise advice}

The TFAS is a web application that was developed by the Health Science and Physical Education Department at TMDU. This application is a life management tool that assesses the balance of adequate exercise, nutrition, and rest for undergraduate students in education. All participants will be asked to input 
their diet and exercise data per day for a week into the TFAS, the results will be analyzed, and the participants will receive unique dietary and exercise advice on video. The advice sessions will last 30 minutes and be conducted by a specialist (TM).

\section{Treatments in the intervention group}

Fixed or removable dentures will be given to participants in the intervention group if they are missing teeth. The technique used to create the fixed dentures involves preparation, impression, recording of jaw relationships, and delivery of the new denture. For the removable dentures, the technique involves primary and secondary impressions, recording of jaw relationships, trial insertions, and delivery of the new denture. Participants who have periodontitis will also receive non-surgical treatment, including periodontal examination, tooth brushing instruction, scaling, reexamination, and scaling and root planning (and subsequent examination). All dental treatments will be completed within 3 months by four dentists with over 6 years' clinical experience each (MK, YK, MI, HS).

\section{Outcome measures}

Outcome measures will be assessed at baseline and at 1 and 3 months after the interventions by a single dentist (MD). Figure 2 shows the schedule of enrollment, intervention, and assessments following SPIRIT statement. Assessment of objective masticatory function [22,23] will be conducted for participants who receive prosthetic treatment. The outcome assessor will also check the completed questionnaires with participants at the end of each assessment to avoid missing data.

\section{Primary outcome measure}

The primary outcome is the waist circumference based on the definition that visceral fat accumulation is central to MetS [3] and that there is a reported correlation between visceral fat and waist circumference [24]. Waist circumference will be measured at the navel in a standing position.

\section{Secondary outcome measures}

In addition to the primary outcome, various secondary outcomes will also be assessed. These include blood pressure, various blood parameters, anthropometric measurements, health-related and oral-healthrelated quality of life, food and nutrient intake, objective masticatory function, and periodontal state.

Blood pressure. Hypertension is a component of MetS, so blood pressure will be measured. The sphygmomanometer will be level with the heart in a sitting posture.

Blood sample test. Hyperglycemia and dyslipidemia are also components of MetS. Therefore, we will measure serum triglyceride, high-/low-density lipoprotein cholesterol, fasting blood glucose, and HbA1c. All participants will be required to fast for more than 6 hours before collecting a $10 \mathrm{~mL}$ blood sample.

Anthropometric measurements. Weight and body composition will be assessed by bioelectrical impedance (MC-780A; Tanita, Tokyo, Japan). Body composition will then be estimated by determining the 
body fat percentage and skeletal muscle mass.

Health-related quality of life. Health-related quality of life will be assessed using the valid and reliable MOS Short-Form 36-Item Health Survey $[25,26]$. This contains 36 questions that evaluate eight health concepts: vitality, physical functioning, bodily pain, general health perceptions, physical role functioning, emotional role functioning, social role functioning, and mental health. The lower a given score, the greater the disability.

Oral health-related quality of life. This will be assessed using the Oral Health Impact Profile-14 (OHIP-14) [27] and the Geriatric Oral Health Assessment Index (GOHAI) [28]. The OHIP-14 contains 14 questions and seven conceptual subscales: functional limitation, pain, psychological discomfort, physical disability, psychological disability, social disability, and handicap. Higher scores indicate lower oral health-related quality of life. The GOHAl contains 12 questions and three conceptual subscales: functional, psychosocial, and pain/discomfort. Higher scores indicate better oral health-related quality of life.

Food and nutrient intake. The brief-type self-administered diet history questionnaire $[29,30]$ will be used to assess the frequency of intake of 75 food items during the previous month. The daily intake of approximately 30 nutrients and 50 food items is then estimated. The validity and reliability of this questionnaire have been established [29, 30].

Periodontal examination. Periodontal examination will involve assessment of probing pocket depth and bleeding on probing [31].

Objective masticatory function. Participants who receive prosthetic treatment will undergo objective assessment of masticatory function. Mixing ability will be measured using a chewing gum (Xylitol Masticatory Performance Evaluating Gum; Lotte Co., Ltd., Tokyo, Japan) that gradually changes its color from green (before mastication) to red during mastication [22]. Participant will chew the gum 60 times at the rate of 1 chew per second. Mixing ability will be calculated based on a Masticatory Performance Index (MPIG) provided with the color-changeable chewing gum, measured by colorimetry (CR-13; KonicaMinolta Sensing, Tokyo, Japan) [32] and the color scale method [33]. Shearing ability will be measured by a test gummy jelly (UHA Mikakuto Co., Ltd., Osaka, Japan) [23]. Participants will chew this gummy jelly 30 times with no restrictions and shearing ability will be measured by a visual scoring method [34].

\section{Sample size estimation}

The sample size has been estimated based on the need to find significant data for the primary outcome, waist circumference. We calculated that 80 participants (40 per group) will be required to achieve an $80 \%$ power to detect a difference of $3.0 \mathrm{~cm}(S D: 5.0 \mathrm{~cm})$ in waist circumference, with a two-sided alpha level of 0.05 . This is based on the assumption that abdominal circumferences are normally distributed [35]. Allowing for a $20 \%$ drop-out rate, we will recruit 100 participants (50 per group).

\section{Randomization and blinding}


Eligible study participants are to be alternately and randomly assigned to either the dietary intervention group or the control group. The randomization sequence has been established with block sizes of 10 and an allocation ratio of 1:1 and will be concealed in opaque consecutively numbered envelopes, and all randomization processes will be conducted by a dentist (MD). Clinicians cannot be blinded to the allocated group, because the dental intervention will be conducted for the participants of the intervention group only during the trial period. Participants can be blinded to their allocated group because this is not essential to their treatment.

\section{Statistical analysis}

Differences between two groups at each assessment point will be compared by Student's t-tests. Changes within each group between the assessment points will be compared by Tukey's test. Moreover, sub-group analysis will be performed by the results of masticatory function and periodontal examination.

\section{Discussion}

Several randomized controlled trials have revealed that combining prosthetic treatment with dietary advice could improve the intake of fruits, vegetables, and key nutrients in edentulous patients $[12,13,14]$. The reduced intakes of these dietary essentials was also shown to be associated with the onset of lifestyle-related diseases, such as cardiovascular disease and diabetes mellitus $[5,6]$. Moreover, it has been reported that carbohydrate, protein, vitamin ( $A, C, E$, and $K$ ), and mineral (calcium, zinc, and magnesium) intakes are significantly lower among patients with MetS than in health subjects [36]. Periodontitis is also known to be progressed by inflammatory cytokines, such as tumor necrosis factor-a, which are present in obesity. The lipopolysaccharide produced in periodontitis also increases levels of tumor necrosis factor-a, interleukin-6, and C-reactive protein. Levels of these inflammatory cytokine worsen further in cases with comorbid diabetes mellitus, arteriosclerosis, and cardiovascular disease [37]. These reports suggest that combining prosthetic or periodontal treatment with dietary and exercise advice could improve the outcomes of patients with MetS.

The primary outcome of this trial is the waist circumference after follow-up, which will be used as a surrogate measure of change in MetS risk. Because of the known association with visceral fat accumulation [3], it may be more appropriate to measure visceral fat levels precisely by computed tomography (CT). However, CT not only increases radiation exposure but also may add little value over waist circumference. It has been reported, for example, that the visceral fat levels on CT correlate with the waist circumference [24]. Therefore, waist circumference should be an appropriate measure for evaluating the primary outcome.

Another potential issue is that trial participants must meet the Japanese waist circumference requirement for MetS, yet we will not consider other diagnostic variables on which visceral fat accumulation is based, including serum triglyceride levels, serum glucose levels, or blood pressure [3]. Therefore, not all of the included participants will have a diagnosis of MetS, which will be an important limitation. Given that the 
components of MetS are caused by visceral fat accumulation [3], however, a decrease in waist circumference after our intervention may suggest its efficacy for the prevention of MetS.

The outcome measures will be assessed at baseline and at 1 and 3 months after the interventions. Although it may be preferable to investigate the long-term effects, such as those at 6 or 12 months, we are concerned about the needs of providing prosthetic or periodontal treatment to the control group at the end of trial. To investigate these long-term effects would require that participants in the control group not receive necessary prosthetic or periodontal treatment for longer than can be ethically justified. Therefore, we will limit out outcome assessments to 3 months after treatment, at which time participants in the control group will receive prosthetic or periodontal treatment. However, this will lead to problems of its own, as discussed next.

All trial participants will be older than 40 years and will meet the Japanese waist circumference requirement for MetS (male: $\geq 85$, female: $\geq 90$ ) [18]. In the Japanese Survey of Dental Diseases 2016 [38], it was reported that $>30 \%$ of subjects older than 40 years had missing teeth and that $>50 \%$ of subjects older than 55 years had dental prostheses. The same survey also reported that $>40 \%$ of subjects older than 40 years had moderate periodontitis. Given these data, many participants in this trial may need prosthetic and periodontal treatment, which will make it difficult to complete treatment plans within the 3 month time frame that we have stipulated. Therefore, we plan to conduct either prosthetic treatment for the participants with missing teeth or periodontal treatment for the participants without missing teeth, not both, in this trial.

Finally, the dental interventions in this trial will be conducted by four dentists (general practitioners) who each have at least 6 years' clinical experience (MK, YK, MI, and HS). In Japan, periodontists and general practitioners offer these non-surgical periodontal treatments. Therefore, it is appropriate that these professionals deliver such treatment to participants who have periodontitis.

In conclusion, we expect to establish a new preventive method for MetS that can be delivered by dental practitioners. Indeed, the dental consultation room may be an ideal setting to provide advice on diet or exercise [39]. If we can show that combining dental interventions with diet and exercise advice is effective, it may be possible to improve the outcomes of MetS, prevent cardiovascular disease, and extend healthy life expectancy.

\section{Trial Status}

The protocol version number is the first version (March 11, 2019). Participant recruitment began form December 3, 2016, and it will be completed in May 1, 2019, approximately. The baseline outcome assessments, and interventions were ongoing at the time of submission (March 11, 2019).

\section{List Of Abbreviations}


CONSORT, Consolidated Standards of Reporting Trials; CT, Computed tomography; GOHAl, Geriatric Oral Health Assessment Index; HbA1c, Hemoglobin A1c; MetS, Metabolic Syndrome; OHIP-14, Oral Health Impact Profile-14; MPIG, Masticatory Performance Index applicable to color-changeable chewing gum; TFAS, Total Fitness Analysis System; TMDU, Tokyo Medical and Dental University; UMIN, University Hospital Medical Information Network.

\section{Declarations}

\section{Ethics approval and consent to participate}

The study has been approved by the institutional ethics committee of TMDU (no. D2016-028). All participants will be required to provide informed consent before participation.

\section{Consent for publication}

Not applicable.

\section{Availability of data and materials}

Not applicable.

\section{Competing interests}

The authors declare that they have no competing interests.

\section{Funding}

The research is supported by a Grant-in-Aid for Scientific Research (no. 16H05521).

\section{Authors' contributions}

HS (clinician) contributed to the dental interventions, data analysis, and drafting of the manuscript. MK, $\mathrm{YK}$, and $\mathrm{MI}$ (clinicians) contributed to the study design, the dental interventions, and the data analysis. MD (outcome assessor) contributed to the study design, randomization and allocation, and data collection and analysis. TM contributed to the dietary and exercise advice. MS and KO contributed to the recruitment of participants. All authors have approved the final version of the manuscript. YM and SM are responsible for the final approval to submit this manuscript for publication.

\section{Acknowledgments}

Not applicable.

\section{References}

1. Reducing Risks, Promoting Health Life: The World Health Report. World Health Organization, 2002. (accessed November 12, 2018 https://www.who.int/whr/2002/en/) 
2. Ministry of Health, Labour and Welfare Trends in death rates for leading causes of death. (accessed November 12, 2018 https://www.mhlw.go.jp/english/wp/wp-

w4/dl/general_welfare_and_labour/P10.pdf).

3. Matsuzawa Y, Funahashi T, Nakamura T. The concept of metabolic syndrome: contribution of visceral fat accumulation and its molecular mechanism. J Atheroscler Thromb. 2011;18:629-39.

4. Nowjack-Raymer RE, Sheiham A. Numbers of natural teeth, diet, and nutritional status in US adults. J Dent Res. 2007;86:1171-5.

5. Nakamura K, Nagata C, Oba S, Takatsuka N, Shimizu H. Fruit and vegetable intake and mortality from cardiovascular disease are inversely associated in Japanese women but not in men. J Nutr. 2008;138:1129-34.

6. Li M, Fan Y, Zhang X, Hou W, Tang Z. Fruit and vegetable intake and risk of type 2 diabetes mellitus: meta-analysis of prospective cohort studies. BMJ Open. 2014;4:e005497.

7. Patel MH, Kumar JV, Moss ME. Diabetes and tooth loss: an analysis of data from the National Health and Nutrition Examination Survey, 2003-2004. J Am Dent Assoc. 2013;144:478-85.

8. Schwahn C, Polzer I, Haring R, Dörr M, Wallaschofski H, Kocher T, et al. Missing, unreplaced teeth and risk of all-cause and cardiovascular mortality. Int J Cardiol. 2013;167:1430-7.

9. Zhu Y, Hollis JH. Associations between the number of natural teeth and metabolic syndrome in adults. J Clin Periodontol. 2015;42:113-20.

10. Iwasaki M, Sato M, Minagawa K, Manz MC, Yoshihara A, Miyazaki H. Longitudinal relationship between metabolic syndrome and periodontal disease among Japanese adults aged $\geq 70$ years: the Niigata Study. J Periodontol. 2015;86:491-8.

11. Kaye EK, Chen N, Cabral HJ, Vokonas P, Garcia RI. Metabolic syndrome and periodontal disease progression in men. J Dent Res. 2016; 95:822-8.

12. Bradbury J, Thomason JM, Jepson NJ, Walls WG, Allen PF, Moynihan PJ. Nutrition counseling increases fruit and vegetable intake in the edentulous. J Dent Res. 2006;85:463-8.

13. Amagai N, Komagamine $Y$, Kanazawa M, Iwaki M, Jo A, Suzuki H, et al. The effect of prosthetic rehabilitation and simple dietary counseling on food intake and oral health related quality of life among the edentulous individuals: A randomized controlled trial. J Dent. 2017;65:89-94.

14. Suzuki H, Kanazawa M, Komagamine $\mathrm{Y}$, Iwaki M, Jo A, Amagai N, et al. The effect of new complete denture fabrication and simplified dietary advice on nutrient intake and masticatory function of edentulous elderly: A randomized-controlled trial. Clin Nutr. 2018;37:1441-7. 
15. Hayashi J, Hasegawa A, Hayashi K, Suzuki T, Ishii M, Otsuka H, et al. Effects of periodontal treatment on the medical status of patients with type 2 diabetes mellitus: a pilot study. BMC Oral Health.

2017;17:77.

16. Mizuno H, Ekuni D, Maruyama T, Kataoka K, Yoneda T, Fukuhara D, et al. The effects of non-surgical periodontal treatment on glycemic control, oxidative stress balance and quality of life in patients with type 2 diabetes: A randomized clinical trial. PLoS One. 2017;12:e0188171.

17. Schulz KF, Altman DG, Moher D, CONSORT Group. CONSORT 2010 statement: updated guidelines for reporting parallel group randomised trials. Int J Surg. 2011;9:672-7.

18. Matsuzawa Y. Metabolic syndrome--definition and diagnostic criteria in Japan. J Atheroscler Thromb. $2005 ; 12: 301$.

19. Eichner K. Renewed examination of the group classification of partially edentulous arches by Eichner and application advices for studies on morbidity statistics. Stomatol DDR. 1990;40:321-5.

20. Highfield J. Diagnosis and classification of periodontal disease. Aust Dent J. 2009;54:S11-26.

21. Mizuno T, Tai K. Development and examination of the total fitness analysis system (TFAS) for fitness education for university students. Japan Journal for Health, Physical Education, Recreation and Dance in Universities 2013;10:31-40.

22. Hama Y, Kanazawa M, Minakuchi S, Uchida T, Sasaki Y. Properties of a color-changeable chewing gum used to evaluate masticatory performance. J Prosthodont Res. 2014;58:102-6.

23. Ikebe K, Morii K, Matsuda K, Hazeyama T, Nokubi T. Reproducibility and accuracy in measuring masticatory performance using test gummy jelly. Prosthodont Res Pract. 2005;4:9-15.

24. Hiuge-Shimizu A, Kishida K, Funahashi T, Ishizaka Y, Oka R, Okada M, et al. Absolute value of visceral fat area measured on computed tomography scans and obesity-related cardiovascular risk factors in large-scale Japanese general population (the VACATION-J study). Ann Med. 2012;44:82-92.

25. Fukuhara S, Bito S, Green J, Hsiao A, Kurokawa K. Translation, adaptation, and validation of the SF-36 Health Survey for use in Japan. J Clin Epidemiol. 1998;51:1037-44.

26. Fukuhara S, Ware JE Jr, Kosinski M, Wada S, Gandek B. Psychometric and clinical tests of validity of the Japanese SF-36 Health Survey. J Clin Epidemiol. 1998;51:1045-53.

27. Motallebnejad M, Hadian H, Mehdizadeh S, Hajiahmadi M. Validity and reliability of the Persian version of the oral health impact profile (OHIP)-14. Caspian J Intern Med. 2011;2:314-20.

28. Naito M, Suzukamo Y, Nakayama T, Hamajima N, Fukuhara S. Linguistic adaptation and validation of the General Oral Health Assessment Index (GOHAI) in an elderly Japanese population. J Public Health 
Dent. 2006;66:273-5.

29. Kobayashi S, Murakami K, Sasaki S, Okubo H, Hirota N, Notsu A, et al. Comparison of relative validity of food group intakes estimated by comprehensive and brief-type self-administered diet history questionnaires against 16 d dietary records in Japanese adults. Public Health Nutr. 2011;14:1200-11.

30. Kobayashi S, Honda S, Murakami K, Sasaki S, Okubo H, Hirota N, Notsu A, Fukui M, Date C. Both comprehensive and brief self-administered diet history questionnaires satisfactorily rank nutrient intakes in Japanese adults. J Epidemiol. 2012;22:151-9.

31. Pihlstrom BL. Periodontal risk assessment, diagnosis and treatment planning. Periodontol 2000. 2001;25:37-58.

32. Hama Y, Hosoda A, Komagamine Y, Gotoh S, Kubota C, Kanazawa M, et al. Masticatory performancerelated factors in preschool children: Establishing a method to assess masticatory performance in preschool children using colour-changeable chewing gum. J Oral Rehabil. 2017;44:948-56.

33. Hama Y, Kanazawa M, Minakuchi S, Uchida T, Sasaki Y. Reliability and validity of a quantitative color scale to evaluate masticatory performance using color-changeable chewing gum. J Med Dent Sci. 2014;61:1-6.

34. Nokubi T, Yoshimuta Y, Nokubi F, Yasui S, Kusunoki C, Ono T, et al. Validity and reliability of a visual scoring method for masticatory ability using test gummy jelly. Gerodontology 2013;30:76-82.

35. Munakata M, Honma H, Akasi M, Araki T, Kawamura T, Kubota M, et al. Repeated counselling improves the antidiabetic effects of limited individualized lifestyle guidance in metabolic syndrome: JSTOP-METS final results. Hypertens Res. 2011;34:612-6.

36. Al-Daghri NM, Khan N, Alkharfy KM, Al-Attas OS, Alokail MS, Alfawaz HA, et al. Selected dietary nutrients and the prevalence of metabolic syndrome in adult males and females in Saudi Arabia: a pilot study. Nutrients. 2013;5:4587-604.

37. Saito T, Shimazaki Y. Metabolic disorders related to obesity and periodontal disease. Periodontol 2000. 2007;43:254-66.

38. Ministry of Health, Labour and Welfare Survey of Dental Diseases. (accessed November 27, 2018 https://www.mhlw.go.jp/toukei/list/dl/62-28-02.pdf)

39. Harris R, Gamboa A, Dailey Y, Ashcroft A. One-to-one dietary interventions undertaken in a dental setting to change dietary behaviour. Cochrane Database Syst Rev. 2012;3:CD006540.

\section{Figures}




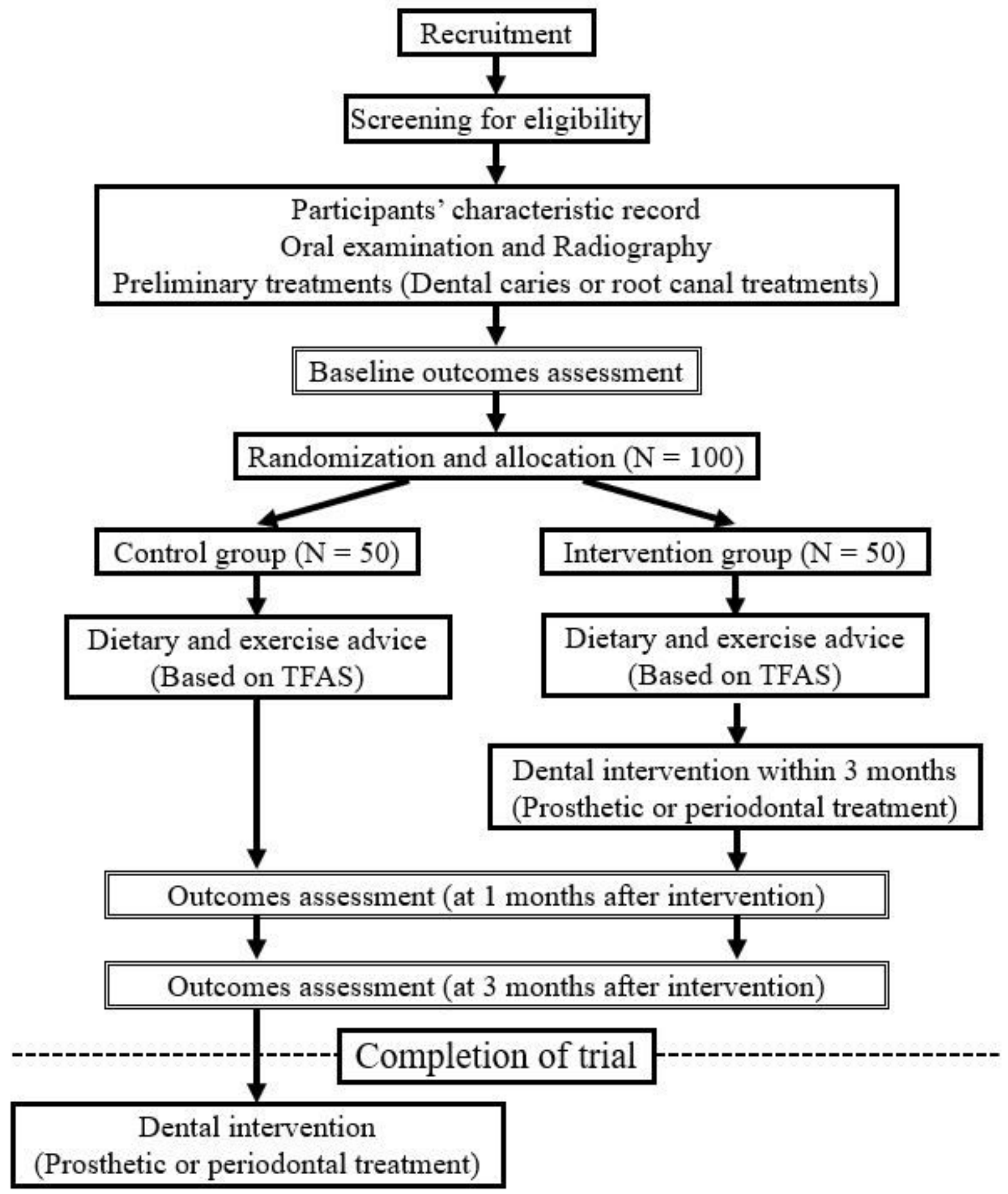

Figure 1

Flow diagram of trial design 


\begin{tabular}{|c|c|c|c|c|c|c|}
\hline & Enrolment & Allocation & \multicolumn{3}{|c|}{ Post-allocation } & Close-out \\
\hline TIMEPOINT** & $-T 1$ & 0 & $\begin{array}{c}T 1 \\
\text { (Baseline) }\end{array}$ & $\begin{array}{c}T 2 \\
\text { (1 month) }\end{array}$ & $\begin{array}{c}T 3 \\
(3 \mathrm{month})\end{array}$ & $T 4$ \\
\hline \hline
\end{tabular}

\section{ENROLMENT:}

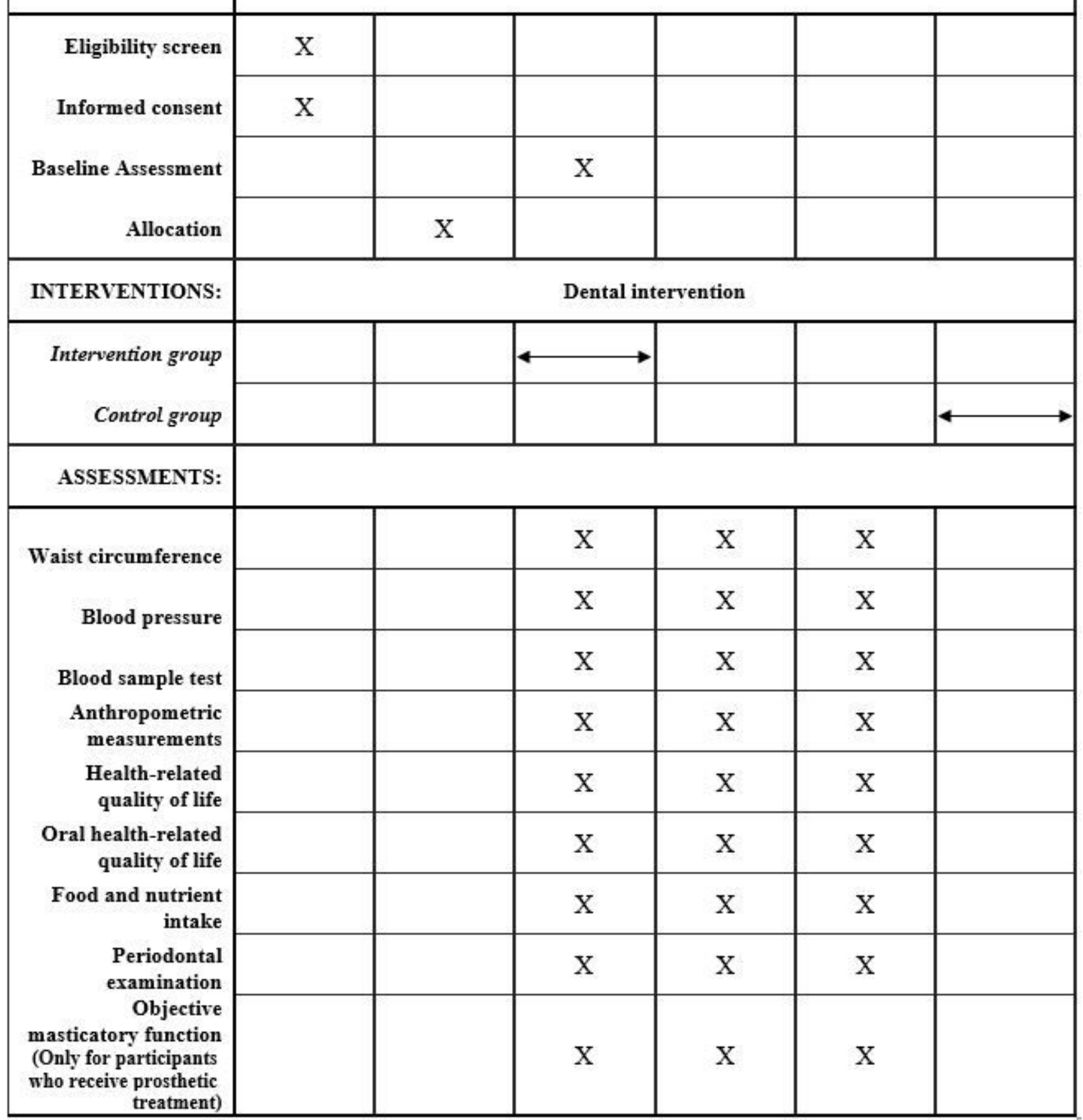

Time of assessment: T1 $=$ baseline, $\mathrm{T} 2=1$ month, $\mathrm{T} 3=3$ months

Figure 2

Schedule of enrollment, intervention, and assessments

\section{Supplementary Files}

This is a list of supplementary files associated with this preprint. Click to download. 
- SPIRITChecklist20190211.doc

Page 16/16 